\title{
Caracterização Físico-Mecânica de Filmes de Borracha Natural Epoxidada Curáveis em Temperatura Ambiente
}

\author{
Rosane M. Jorge \\ Instituto de Macromoléculas Professora Eloisa Mano (IMA), UFRJ \\ Centro Tecnológico do Exército \\ Marcos T. Ferreira \\ Laboratório de Química Militar, Centro Tecnológico do Exército \\ Paulo H. S. Picciani, Ailton S. Gomes, Regina C. R. Nunes \\ Instituto de Macromoléculas Professora Eloisa Mano (IMA), UFRJ
}

\begin{abstract}
Resumo: Neste trabalho é apresentado o estudo da formação de reticulações na temperatura ambiente, de borracha natural epoxidada (ENR) de $25 \%$ de grau de epoxidação, com um agente de cura contendo o tiol trimetilolpropano tris(2-mercaptoacetato) (TMP-SH). As composições foram realizadas a partir de solubilização da ENR e, posterior reação com o TMP-SH. Foram preparadas composições com 5, 10 e 20 phr de ENR e controles contendo NR e ENR. Também foi preparada uma composição com NR e $10 \mathrm{phr}$ de TMP-SH para comprovar o efeito do grupo epóxido. A formação de reticulação dos filmes foi determinada pelas propriedades físico-mecânicas como grau de inchamento no equilíbrio, resistência à tração e propriedades dinâmico-mecânicas. Os resultados mostraram a eficiência do agente de cura e a influência do teor incorporado de TMP-SH nas propriedades determinadas, e a composição de ENR com 10 phr do agente de cura foi a que apresentou melhor desempenho físico-mecânico.
\end{abstract}

Palavras-chave: Borracha natural epoxidada, cura, resistência à tração, DMA, grau de inchamento.

\section{Physical-Mechanical Characterization of Epoxidized Natural Rubber Films Curable at Ambient Temperature}

Abstract: This paper presents the crosslinking study at room temperature of epoxidised natural rubber (ENR), of 25\% of epoxidation level, with a curing agent containing thiol trimethylolpropane tris(2-mercaptoacetate) (TMP-SH). The compositions were made from solubilization of ENR, and subsequent reaction with TMP-SH. Compositions with 5, 10 and $20 \mathrm{phr}$ of TMP-SH, NR and ENR controls were prepared. It was also prepared a composition with NR and 10 phr of TMP-SH to demonstrate the effect of epoxide group. The crosslinking formation of the films was determined by physicalmechanical properties as equilibrium swelling index, tensile strength and dynamic-mechanical properties. The results showed the efficiency of the cure agent and the influence of TMP-SH content incorporated, in the studied properties, and the ENR composition with $10 \mathrm{phr}$ of curing agent presented the best physical-mechanical performance.

Keyword: Epoxidized natural rubber, cure, tensile strength, DMA, swelling index.

\section{Introdução}

Revestimentos especiais para uso como material absorvedor de gás hidrogênio podem ter aplicações civis e militares. Eles são colocados em contato com materiais que liberam gás hidrogênio em espaços fechados. Uma das aplicações é o uso como material de proteção de artefatos pirotécnicos contendo metais ativos que ao se degradarem liberam gás hidrogênio. O material é utilizado como um revestimento no próprio artefato pirotécnico ou dentro das embalagens, para o armazenamento e transporte dos mesmos $^{[1,2]}$. Este tipo de revestimento deve ser composto por uma matriz polimérica com alto conteúdo de insaturações, com possibilidade de ser curado em temperaturas próximas a ambiente, e preferencialmente ser feito com matériasprimas disponíveis no mercado nacional.É desejável também que estes revestimentos sejam de fácil aplicação e tenham resistência estrutural para o manuseio e transporte ${ }^{[1,2]}$.

Com o crescente interesse na exploração de fontes renováveis de matérias-primas para o desenvolvimento de

Autor para correspondência: Rosane M. Jorge, Universidade Federal do Rio de Janeiro, Av. Horacio Macedo, 2030, Centro de Tecnologia, Bloco J, 
novos materiais, a borracha natural (NR) foi escolhida como matriz no desenvolvimento de formulações para revestimento neste trabalho, pois além de ser de fácil aquisição em nosso país, tem baixo custo e elevadas propriedades de desempenho ${ }^{[3,4]}$, se adequando ao estudo, pelo grande número de duplas ligações na cadeia polimérica, mesmo após cura.

A epoxidação é um método conhecido e versátil para transformação das propriedades físicas limitantes da borracha natural, tais como, a resistência a óleo, ao oxigênio e ao ozônio ${ }^{[5,6]}$. Uma propriedade importante em revestimentos é a aderência, que é aumentada pela polaridade de acordo com a extensão da epoxidação introduzida ${ }^{[5]}$. Neste trabalho a NR foi modificada com a introdução de grupos epóxidos para o desenvolvimento de revestimentos, com potencial de utilização em artefatos pirotécnicos.

O grupo oxirano (epóxido) é muito reativo e é considerado um dos mais importantes intermediários em síntese orgânica. A borracha natural epoxidada (ENR) pode sofrer a reação de cura tanto pelas duplas ligações, pela vulcanização tradicional com enxofre, como também pelos grupos oxiranos ${ }^{[5]}$. Além disso, na literatura é descrito que o grupo epóxido é o responsável pela interação da ENR, aumentando a compatibilidade entre carga-polímeros ${ }^{[7-9]}$ ou em misturas físicas ${ }^{[10,11]}$, e também em sistemas contendo agente de reticulação e alta energia (radiação UV, feixe de elétrons, etc. $)^{[12-14]}$.

Para a aplicação como revestimento, a borracha natural epoxidada foi solubilizada em tolueno, para o preparo da composição curável a temperatura ambiente, pela adição do agente de cura sintetizado. Neste trabalho o agente de cura foi o trimetilolpropano tris(2-mercaptoacetato) (TMP-SH) devido às suas características químicas como não ter enxofre elementar ${ }^{[2]}$, ter reatividade em relação ao epóxido da $\operatorname{ENR}^{[5]}$ e também por reagir em temperaturas mais baixas do que a da cura tradicional $\left(140{ }^{\circ} \mathrm{C}\right)^{[3]}$. Como o grupo epóxido reage com muita facilidade com grupos tióis ${ }^{[15]}$, foi proposto neste trabalho o uso do TMP-SH para reagir com a ENR, formando reticulações e levando à cura da ENR em temperatura ambiente. Os filmes obtidos com ENR foram analisados quanto às propriedades físico-mecânicas como grau de inchamento no equilíbrio, resistência à tração e propriedades dinâmico-mecânicas.

\section{Experimental}

\section{Materiais}

Neste trabalho foi utilizado o látex de borracha natural (NR) com alta concentração de amônia, contendo $60 \%$ de borracha seca (DRC) (ABNT NBR 11598) ${ }^{[16]}$, do seringal Xapuri, Acre, e fornecido pela Teadit Indústria e Comércio Ltda. O surfatante não iônico utilizado para estabilizar o látex de NR foi o Ultranex NP 95, nonil fenil poli(etileno glicol), fornecido pela Oxiteno, com 9,5 mols de etileno glicol (EO) e balanço hidrofílico-lipofísico (HLB) de 13. Os demais produtos químicos usados neste trabalho foram os seguintes: ácido fórmico $98 \%$, peróxido de hidrogênio $30 \%$, etanol absoluto P.A., e tolueno P.A. da Vetec Quimica Fina; ácido tioglicólico 99\% P.A da Isofar, e trimetilolpropano (TMP) 98\% G.C da Sigma-Aldrich.

Trimetilolpropano tris(2-mercaptoacetato) (TMP-SH) foi sintetizado em laboratório, pela esterificação direta do TMP com ácido tioglicólico.

\section{Reação de epoxidação}

A borracha natural epoxidada (ENR), com 25\% de grupamento epóxido, foi sintetizada em laboratório, a partir do látex de NR estabilizado com surfatante, utilizando perácido orgânico gerado in situ (com ácido fórmico e peróxido de hidrogênio $)^{[17,18]}$. A reação foi realizada em uma dada temperatura e com o tempo de reação necessário para obtenção do grau de epoxidação desejado. Após o término da reação o látex epoxidado foi neutralizado, coagulado e seco. O teor de epoxidação da ENR foi determinado por ${ }^{1} \mathrm{HNMR}^{[19]}$. A reação de epoxidação da borracha natural é apresentada na Figura 1.

\section{Preparação dos filmes de ENR curáveis em temperatura ambiente}

Inicialmente ENR seca foi dissolvida em tolueno até total solubilização $(5 \% \mathrm{p} / \mathrm{v})$, sendo posteriormente preparadas composições com 5, 10 e 20 phr (parte por 100 partes de borracha) do agente de cura TMP-SH, com agitação magnética à temperatura ambiente. A reação da borracha natural epoxidada com o tiol foi mantida por 24 horas, para a incorporação dos grupos tióis nas cadeias da ENR. A solução resultante foi vazada para um recipiente de superfície plana e submetida à evaporação lenta e controlada, finalizando com a secagem dos filmes a vácuo, na temperatura ambiente, até peso constante ${ }^{[20]}$. Após a secagem os filmes se tornavam insolúveis, comprovando a cura da borracha. Para a comprovação do efeito do grupo epóxido foi feita uma composição de NR não epoxidada com o agente reticulante TMP-SH no teor de $10 \mathrm{phr}$ e após secagem os filmes solubilizaram em tolueno. Também foram preparadas duas formulações para controle, com um único componente, uma com NR e outra com ENR puras, para efeito de comparação. As formulações são apresentadas na Tabela 1. Todos os filmes eram transparentes.

\section{Propriedades físico-mecânicas}

\section{Grau de inchamento}

O inchamento no equilíbrio dos filmes de ENR foi determinado em tolueno, sob condições ambientes ${ }^{[21]}$. Corpos de prova com as dimensões de $10 \times 10 \times 0,7 \mathrm{~mm}$ foram cortados dos filmes preparados, e em seguida foram imersos no solvente a $0,5 \%$ w/v e deixados no escuro. De tempos em tempos as amostras eram retiradas do solvente, secas em papel de filtro para remover o excesso de solvente da superfície, pesadas, e então recolocadas no solvente até 


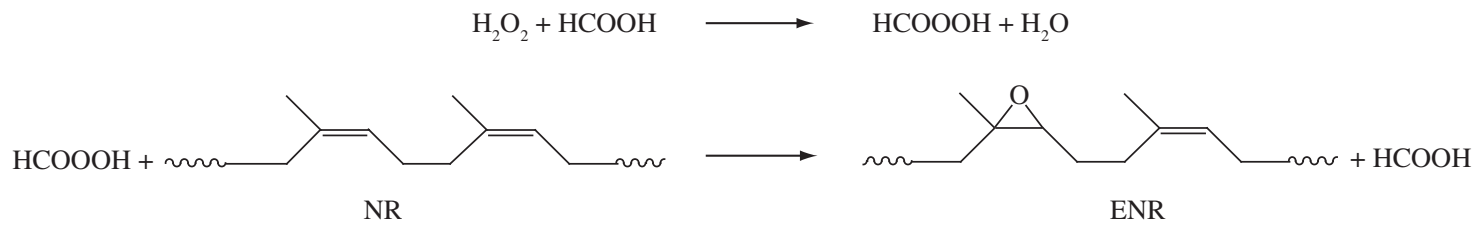

Figura 1. Reação de epoxidação da borracha natural.

Tabela 1. Formulações dos filmes ENR e NR com TMP-SH e dos controles (NR e ENR).

\begin{tabular}{|c|c|c|c|c|c|c|}
\hline \multirow[t]{2}{*}{ Materiais } & \multicolumn{6}{|c|}{ Quantidades (phr) $^{\mathrm{a}}$} \\
\hline & NR & NR 10 & ENR & ENR 5 & ENR 10 & ENR 20 \\
\hline NR & 100 & 100 & - & - & - & - \\
\hline ENR & - & - & 100 & 100 & 100 & 100 \\
\hline TMP-SH & - & 10 & - & 5 & 10 & 20 \\
\hline
\end{tabular}

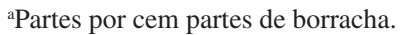

o inchamento no equilíbrio. Os corpos de prova atingiram o inchamento no equilíbrio em até 24 horas, com exceção dos controles (NR e ENR) que dissolveram em poucas horas de teste e da composição NR 10 que ficou parcialmente solúvel. O resultado para cada amostra foi obtido a partir do valor médio de três determinações. O porcentual em grau de inchamento $\alpha$, foi calculado usando a seguinte Equação 1:

$$
\alpha=\frac{\left(W_{f}-W_{i}\right)}{W_{i}} \times 100
$$

onde $\mathrm{W}_{\mathrm{f}}$ é o peso final e $\mathrm{W}_{\mathrm{i}}$ é o peso inicial, em $\mathrm{g}$.

\section{Resistência à tração}

As propriedades de resistência à tração dos filmes foram determinadas segundo norma DIN 53504 ${ }^{[22]}$, utilizando a máquina de testes universal EMIC DL 3000 com célula TRD 22, velocidade das garras de $200 \mathrm{~mm} / \mathrm{min}$ e temperatura ambiente, com corpos de prova tipo S2 extraídos de filmes vasados $^{[20]}$. Foram utilizados 5 corpos de prova de cada composição, e os resultados dos ensaios foram registrados como mediana dos valores parciais.

\section{Propriedades dinâmico-mecânicas}

A análise dinâmico-mecânica (DMA) dos filmes foi realizada em analisador DMA Q $800 \mathrm{~V} 7.5$ da marca TA Instruments. Filmes retangulares de 18,6 × 6,5 × 0,7 mm (comprimento, largura, espessura) foram analisados em modo de deformação de tensão. Os experimentos foram realizados em frequência de $1 \mathrm{~Hz}$, com velocidade de aquecimento de $2{ }^{\circ} \mathrm{C} /$ min, faixa de temperatura de -80 a $40{ }^{\circ} \mathrm{C}$ e deformação de $0,01 \%$. Módulo de armazenamento (G'), módulo de perda $\left(G^{\prime \prime}\right)$ e tangente de perda $\left(\tan \delta=G^{\prime \prime} / G^{\prime}\right)$ foram determinados para todas as amostras sob idênticas condições.

\section{Resultados e Discussão}

A reação proposta entre a ENR e o TMP-SH está ilustrada na Figura 2. A reação ocorre entre o grupo tiol $(\mathrm{SH})$ do TMP-SH e o epóxido da ENR. Posteriormente os dois outros grupos tióis das ramificações do TMP-SH, reagem com os grupos epóxidos de uma cadeia adjacente de ENR, deste modo levando a formação de reticulação.

A Tabela 2 mostra os resultados do ensaio de resistência à tração e do grau de inchamento no equilíbrio para os filmes preparados com ENR e NR e das borrachas puras.

Os resultados mecânicos da NR comparados com os da NR 10 (Tabela 2) mostram a pequena influencia do agente de cura no aumento do desempenho mecânico, tornando a borracha menos elástica, conforme o resultado de alongamento na ruptura.

Os resultados da Tabela 2 mostram que a adição do TMP-SH na matriz de borracha modifica o comportamento físico-mecânico da ENR, podendo ser atribuído a reação do agente de cura trifuncional com a ENR. Os resultados mais baixos de todas as determinações foram para a borracha natural epoxidada (ENR), seguida da borracha natural não epoxidada (NR), como era de se esperar, pela ausência do agente de cura. Aumentando o teor de TMP-SH nas composições, os valores de módulo a $100 \%$ e tração na ruptura aumentam até o valor de $10 \mathrm{phr}$ do TMP-SH. Estes resultados estão relacionados ao número de reticulação mais indicado para esta composição, pois seguindo $\operatorname{Coran}^{[23]}$ a resistência à tração é dependente da quantidade de reticulação. A rigidez molecular pode ser comprovada também pela diminuição do alongamento na ruptura na composição com $10 \mathrm{phr}$ de agente de cura $^{[14]}$, bem como pela menor energia de ruptura, entre as composições com TMP-SH.

Desta forma é possível escolher entre as composições desenvolvidas aquela que mais se adequa a finalidade proposta, em relação ao desempenho mecânico, que foi a ENR10.

O grau de inchamento também é função da introdução do grupo epóxido na NR, porque reduz a flexibilidade da cadeia, diminuindo assim o inchamento em solventes. A redução da flexibilidade da cadeia é devido à estrutura do epóxido e também à polaridade que aumenta as interações entre as cadeias ${ }^{[24]}$. Porém, neste trabalho o teor de epóxido foi mantido constante em todas as composições desenvolvidas.

O aumento da adição no teor de TMP-SH nas composições fez diminuir o grau de inchamento em tolueno das composições com ENR o que leva a um aumento no teor de gel, sendo um indicativo de formação de reticulações entre ENR e TMP-SH em função do teor do agente de cura $^{[12,13]}$. Segundo Tangboriboonrat ${ }^{[25]}$ um valor de grau de inchamento menor do que $500 \%$ pode corresponder a uma borracha reticulada. 

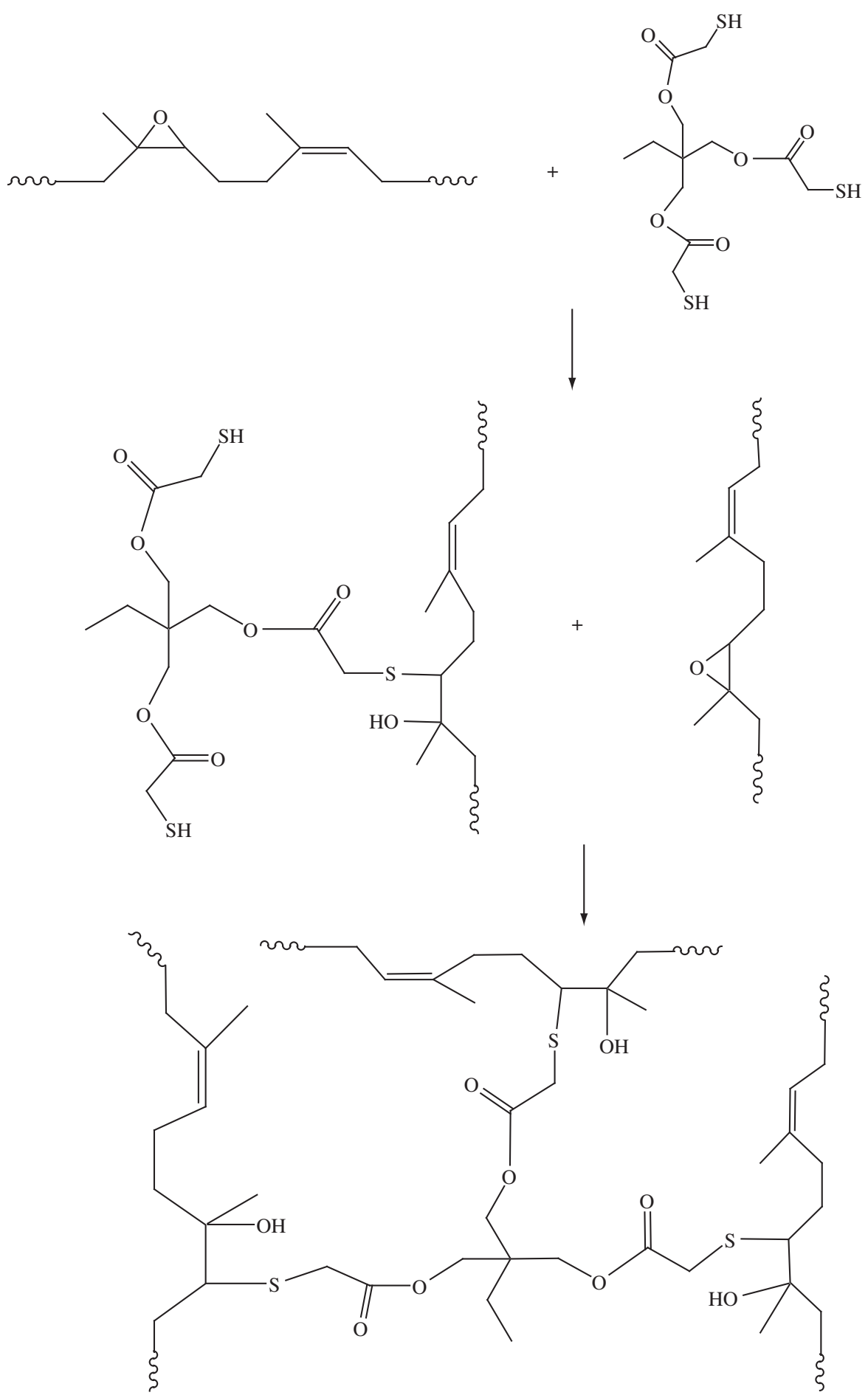

Figura 2. Reações prováveis da borracha natural epoxidada com TMP-SH.

Tabela 2. Resistência à tração e grau de inchamento dos filmes ENR/TMP-SH, NR/TMP-SH e dos controles (NR e ENR).

\begin{tabular}{lcccccc}
\hline \multicolumn{1}{c}{ Resultados/filmes } & NR & NR 10 & ENR & ENR 5 & ENR 10 & ENR 20 \\
\hline Modulo a 100\% (MPa) & $0,3 \pm 0,04$ & $0,6 \pm 0,04$ & $0,20 \pm 0,01$ & $0,6 \pm 0,1$ & $1,1 \pm 0,2$ & $0,4 \pm 0,03$ \\
Tensão na ruptura (MPa) & $0,3 \pm 0,05$ & $0,6 \pm 0,06$ & $0,24 \pm 0,02$ & $1,32 \pm 0,2$ & $1,40 \pm 0,08$ & $0,98 \pm 0,03$ \\
Deformação na ruptura (\%) & $413 \pm 30,7$ & $398 \pm 267,0$ & $400 \pm 162,4$ & $316 \pm 30,0$ & $158 \pm 16,4$ & $321 \pm 21,0$ \\
Energia na ruptura (N.mm) & $134,3 \pm 25,4$ & $134,9 \pm 103,6$ & $71,2 \pm 21,4$ & $115,8 \pm 7,5$ & $92,9 \pm 23,9$ & $120,3 \pm 178,6$ \\
Grau de inchamento (\%) & $\mathrm{S}$ & PS & $\mathrm{S}$ & $639 \pm 7$ & $370 \pm 3$ & $372 \pm 4$ \\
\hline
\end{tabular}

$\mathrm{S}=$ amostra solúvel; e $\mathrm{PS}=$ amostra parcialmente solúvel. 
Pelos resultados da Tabela 2 pode-se concluir que houve reticulação dos filmes de ENR em presença de TMP-SH, diferentemente do que ocorreu nas borrachas puras (NR e ENR) e na composição de NR com 10 phr TMP-SH, o que é uma indicação da reação de reticulação ser preferencialmente feita pelo grupamento epóxido ${ }^{[10]}$. No caso da NR 10, a dissolução desta composição em tolueno mostrou a formação de géis, indicando pouca reticulação entre as cadeias o que pode ser atribuído à menor reatividade da NR não epoxidada em comparação a ENR ${ }^{[26]}$.

Os valores de propriedades físico-mecânica dos filmes preparados neste trabalho estão dentro dos valores mostrados na literatura, em procedimentos de preparação semelhantes ao usado neste trabalho ${ }^{[12,20]}$. Optou-se por esta técnica devido à futura aplicação destes filmes, curáveis a temperatura ambiente, como revestimentos para captação de hidrogênio.

As curvas para módulo de armazenamento $\left(\mathrm{G}^{\prime}\right)$, tangente de perda (Tan $\delta$ - G'/G'), e módulo de perda (G') em função da temperatura, na faixa de -80 a $40{ }^{\circ} \mathrm{C}$, obtidas da análise dinâmico-mecânica, dos filmes deNR, ENR, deENR/TMP-SH e de NR/ TMP-SH são mostradas nas Figuras 3a, b e c. A temperatura de transição vítrea $(\mathrm{Tg})$ foi determinada como a temperatura máxima do pico da Tan $\delta^{[27]} \mathrm{e}$ os resultados de maior destaque estão mostrados na Tabela 3.

Segundo Cramer ${ }^{[28]}$, examinando o módulo no regime borrachoso (módulo de armazenamento acima da $\mathrm{Tg}$ ) dos polímeros via DMA, é possível fazer uma estimativa a respeito da formação de reticulado ou não. Os resultados da Tabela 3 e da Figura 3a mostram o aumento do módulo no regime borrachoso $\left(20^{\circ} \mathrm{C}\right)$ influenciado pelo teor do tiol, indicando a formação de reticulação nas composições de ENR, corroborando os resultados de tração na ruptura e de inchamento.

Como mostrado também na Tabela 2, a NR tem melhor desempenho mecânico do que a ENR, e este comportamento se repete, como era de se esperar, na analise do desempenho por DMA destas duas composições no regime borrachoso $\left(20^{\circ} \mathrm{C}\right)$.

Para a composição de NR com 10 phr de TMP-SH o módulo de armazenamento foi reduzido em relação à NR além de reduzir a temperatura de transição vítrea, indicando a formação de pouca reticulação ${ }^{[28]}$. Também é mostrado dois picos na curva de tangente delta em função da temperatura para a composição NR 10 (Figura 3b) indicando um comportamento de duas fases imiscíveis da NR e do TMP-SH ${ }^{[27]}$. Esse resultado está de acordo com aqueles de propriedades mecânicas, podendo concluir a maior reatividade da ENR em relação ao TMP-SH do que a NR.

No caso da $\tan \delta^{[27]}$, quanto mais fina for a largura do pico a meia altura, mais homogênea é a mistura. Neste trabalho a composição ENR 5 apresenta-se a mais homogênea do que a ENR 10 e ENR 20. Para os filmes de ENR/TMP-SH o aumento do teor do agente de cura faz alargar o pico da $\tan \delta^{[13]}$. Nas composições ENR 10 e ENR 20 o alargamento do pico sugere a existência de mais de uma fase ${ }^{[29]}$.

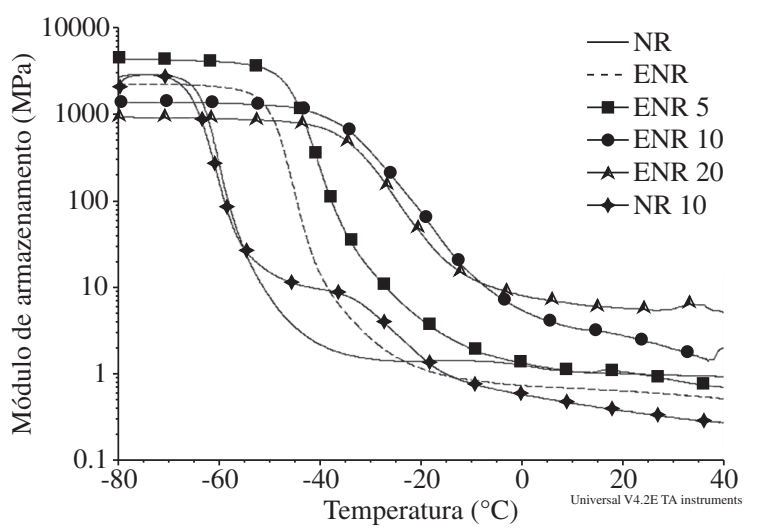

(a)

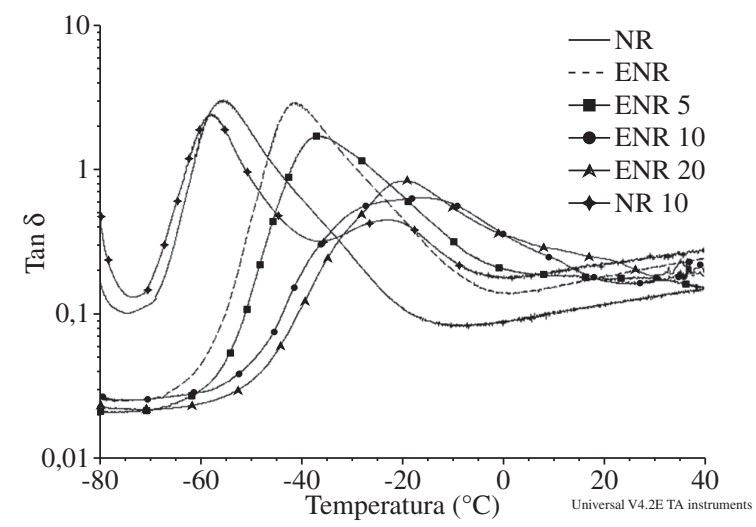

(b)

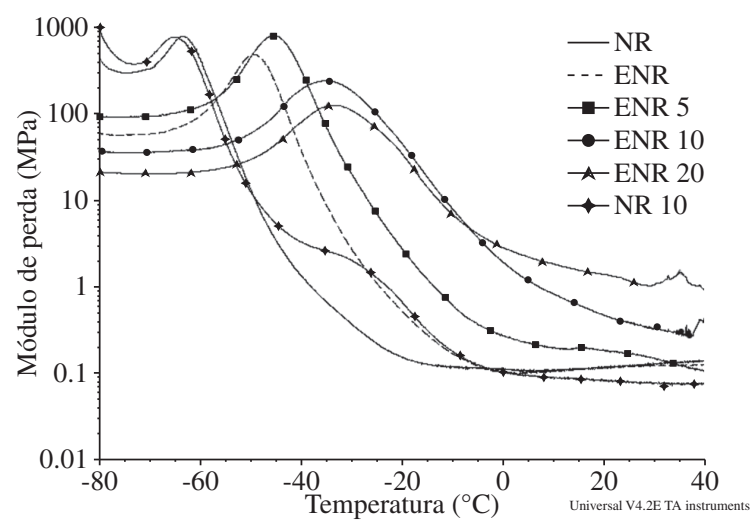

(c)

Figura 3. Resultados dinâmico-mecânicos dos filmes ENR/TMP-SH, NR/TMP-SH e dos controles (NR e ENR) a) Módulo de armazenamento; b) $\tan \delta$; e c) Módulo de perda.

Pelos resultados de DMA pode ser afirmado que houve a formação de maior número de reticulações na ENR com TMP-SH, aumentando a estrutura tridimensional ${ }^{[10]}$, mostrado pelo deslocamento do pico da $\operatorname{Tan} \delta$ para temperaturas mais altas (Figura 3b) ) $^{[12,20,28]}$ e diminuição do valor (da altura pico) (Tabela 3), como consequiência do aumento da rigidez molecular ${ }^{[27]}$. A redução da Tan $\delta$, para o filme de ENR10 e ENR 20 em comparação a NR pura está relacionada à razão entre módulo de perda e de armazenamento (G’/G'). 
Tabela 3. Resultados dinâmico-mecânicos dos filmes ENR/TMP-SH, NR/TMP-SH e dos controles (NR e ENR).

\begin{tabular}{|c|c|c|c|c|c|}
\hline \multirow[t]{2}{*}{$\begin{array}{l}\text { Filmes/ } \\
\text { resultados }\end{array}$} & \multicolumn{2}{|c|}{$\begin{array}{c}\text { Módulo de } \\
\text { armazenamento (MPa) }\end{array}$} & \multicolumn{2}{|c|}{$\operatorname{Tan} \delta(\mathbf{M P a})$} & \multirow[t]{2}{*}{$\begin{array}{l}\mathbf{T g} \\
\left({ }^{\circ} \mathbf{C}\right)\end{array}$} \\
\hline & na Tg & $20^{\circ} \mathrm{C}$ & na Tg & $20^{\circ} \mathrm{C}$ & \\
\hline NR & 31,35 & 1,01 & 3,02 & 0,12 & $-55,50$ \\
\hline NR 10 & 57,04 & 0,37 & 2,4 & 0,22 & $-57,89$ \\
\hline ENR & 21,52 & 0,63 & 2,89 & 0,18 & $-41,47$ \\
\hline ENR 5 & 58,78 & 1,06 & 1,71 & 0,17 & $-36,72$ \\
\hline ENR 10 & 40,62 & 2,77 & 0,64 & 0,17 & $-16,34$ \\
\hline ENR 20 & 40,30 & 5,73 & 0,83 & 0,24 & $-19,77$ \\
\hline
\end{tabular}

Os resultados corroboram com os valores de propriedades mecânicas.

Valores de módulo de perda em função da temperatura dos filmes são mostrados na Figura 1c. O fator de perda é um indicativo do grau de reticulação e do grau de mistura. $\mathrm{Na}$ Figura 1c é mostrado a presença de um único pico que pode também ser relacionado à $\mathrm{Tg}$ dos diferentes filmes para ENR/TMP-SH. Com a ENR pura ocorre o deslocamento do pico de módulo de perda e o valor da Tg para mais altas temperaturas comparado à NR, devido à introdução do grupamento epóxido na $\mathrm{NR}^{[5,27]}$. Apenas para a NR 10 é apresentada duas transições. As mesmas conclusões na análise da Tan $\delta$ podem ser sugeridas para os resultados de módulo de perda.

\section{Conclusões}

Foram obtidos filmes de borracha natural epoxidada, transparentes e curáveis a temperatura ambiente.

$\mathrm{O}$ agente de cura, usado em diferentes teores influenciou os resultados obtidos. A eficiência do agente de cura usado e a formação de reticulações foram comprovadas pela determinação do inchamento no equilíbrio, resistência à tração e análise dinâmico-mecânica.

Foi comprovado o efeito do epóxido sobre o TMP-SH, pela comparação dos resultados das composições de ENR e NR com o agente de cura.

Dentre as composições analisadas, a de melhor desempenho mecânico foi o filme com $10 \mathrm{phr}$ do agente de cura, cujos resultados são conseqüência do maior teor em reticulações, corroborado pela mais elevada temperatura de transição vítrea, e mais baixo valor para o máximo de Tan $\delta$ na temperatura e acima da Tg. Esses resultados também são corroborados pelo grau de inchamento.

Desta forma será possível escolher entre as composições desenvolvidas e com valores próximos de resistência à tração, aquela que mais se adequa a finalidade proposta, em relação à deformação e à rigidez (módulo).

Com base nos resultados obtidos, os futuros trabalhos darão suporte para o uso destes filmes como absorvedores de hidrogênio.

\section{Agradecimentos}

Os autores agradecem aos órgãos de fomento $\mathrm{CNPq}$, CAPES e FUJB pelo apoio financeiro, e a BRENTAG Química Brasil Ltda pelo fornecimento do surfatante, e a TEADIT Indústria e Comércio Ltda pelo fornecimento do látex de NR.

\section{Referências Bibliográficas}

1. Liu, C. Z.; Shi, L. Q.; Xu, S. L.; Zhou, Z. Y.; Luo, S. Z. \& Long, X. G. - Vacuum, 75, p.71 (2004).

2. Posson, P. L. - WO 9963298, Patent Cooperation Treaty (PCT), Walnut Creek, (1999).

3. Bhowmick, A. K.; Hall, M. M. \& Benarey, H. A. - "Rubber Products Manufacturing Technology", Marcel Dekker Inc., New York (1994).

4. Escócio,V. A.; Martins, A. F.; Costa, D. M. R; Visconte, L. L. Y. \& Nunes, R. C. R. - Polím. Ciênc. e Tecnol., 13, p.132 (2003).

5. Gelling, I. R. \& Porter, M. - "Chemical Modification of Natural Rubber" in: Natural rubber science and technology, A. D. Roberts (ed.), Malaysian Rubber Producers Research Association, Oxford University Press, Oxford, 1990.

6. Derouet, D.; Mulder-Houdayer, S. \& Brosse, J. C. - J. Appl. Polym. Sci., 95, p.39 (2005).

7. Rocha, T. L. A. C.; Jacobi, M. M.; Samios, D. \& Schuster, R. H. - Polím. Ciênc. Tecnol., 16, p.111 (2006).

8. Rocha, T. L. A. C.; Jacobi, M. M. \& Schuster, R. H. Polím. Ciênc. e Tecnol., 14, p.377 (2004).

9. Bandyopadhyay, A.; Sakkar, M. D. \& Bhowmick, K - J. Polym. Sci.: Part B Polym. Phys., 43, p.2399 (2005).

10. Okwu, U. N. \& Okieimen, F. E. - Europ. Polym. J., 37, p.2253 (2001).

11. Roychoudhury, A.; De, P. P.; Dutta, N. K.; Roychoudhury, N.; Haidar, B. \& Vidal, A. - Rubb. Chem. Technol., 66, p.230 (1993).

12. Ratnam, C. T.; Kamaruddin, S.; Nasir, M.; Baharin, A. \& Zaman, K. - Europ. Polym. J., 37, p.1667 (2001).

13. Ratnam, C. T.; Nasir, M.; Baharin, A. \& Zaman, K. - J. App. Polym. Sci., 81, p.1925 (2001).

14. Ratnam, C. T.; Kamaruddin, S.; Sivachalam, Y.; Talib, M. \& Yahya, N. - Polym. Test., 25, p.475 (2006).

15. Lee, H. \& Neville, K. - "Handbook of epoxy resins", McGraw-Hill Book Company, New York (1967).

16. Associação Brasileira de Normas Técnicas - ABNT. "NBR 11598: Látex concentrado de borracha natural", Rio de Janeiro, Brasil (1989).

17. Phinyocheep, P.; Phetphaisit, C. W.; Derouet, D.; Campistron, I. \& Brosse, J. C. - J. Appl. Polym. Sci., 95, p.6 (2005). 
18. Sanguansap, K.; Suteewong, T.; Saendee, P.; Buranabunya, U. \& Tangboriboonrat, P. - Polym., 46, p.1373 (2005).

19. Burfield, D. R.; Lim, K. L.; Law, K. S. \& Soon, N. G. Polymer, 25, p.995 (1984).

20. Bandyopadhyay, A.; Sakkar, M. D. \& Bhowmick, K. Rubb. Chem. Techn., 77, p.830 (2004).

21. Flory, P. J. - "Principles of Polymer Chemistry", Cornel University Press, New York (1953).

22. Deutsches Institut für Normung - "DIN 53504: Testing of elastomers, determination of ultimate tensile strength, tensile strength, elongation at failure and stress values by a tensile test", Germany (1994).

23. Coran, A. Y. - Rubb. Chem. and Technol., 68, p.351 (1995).
24. Johnson, T. \& Thomas, S. - Polymer, 41, p.7511 (2000).

25. Tangboriboonrat, P. \& Lerthititrakul, C. - Coll. Polym. Sci., 280, p.1097 (2002).

26. Morton, M. - "Rubber Technology", Van Nostrand Reinhold, New York (1983).

27. Canevarolo, S. V. - "Análise térmica dinâmico-mecânica”, in: Técnicas de Caracterização de Polímeros, Antilever Editora Ltda, São Carlos (2004).

28. Cramer, N. B. \& Bowman, C. N. - J. Polym. Sci: Part A: Polym. Chem., 39, p.3311 (2001).

29. Kallitsis, J. K. \& Kalfoglou, N. K. - J. Appl. Polym. Sci., 37, p.453 (1989).

Enviado: 12/06/09

Reenviado: 28/09/09

Aceito: 01/10/09 